\title{
Economic Consequences of Anti-HCV Treatment of Patients Diagnosed Through Screening in Italy: A Prospective Modelling Analysis
}

\author{
Andrea Marcellusi ${ }^{1,2}$. Claudia Simonelli ${ }^{1}$. Francesco S. Mennini ${ }^{1,2} \cdot$ Loreta A. Kondili $^{3}$ on behalf of PITER \\ Collaborating Group available at http://www.progettopiter.it
}

Accepted: 19 July 2021 / Published online: 12 October 2021

(c) The Author(s) 2021

\begin{abstract}
Aim To evaluate the cost-consequences of the investment for anti-hepatitis C virus (HCV) treatment by the Italian National Health System (NHS) for patients who will be newly diagnosed through active HCV screening, implemented in Italy from 2020.

Methods A previously published Markov model was used to estimate the disease complications avoided and the associated savings over 20 years to treat a standardised population of 10,000 HCV-infected patients diagnosed as a result of screening. Disease progression probabilities and fibrosis stage distribution were based on previously reported data in the literature. Real-life treatment effectiveness and medical expenses for disease management were estimated starting from a representative cohort of HCV-treated patients in Italy (Italian Platform for the Study of Viral Hepatitis Therapies). The breakeven point in time (BPT) was defined as the years required for the initial investment in treatment to be recovered in terms of cumulative costs saved.

Results Over a 20-year time horizon, the treatment of 10,000 standardized patients diagnosed through active HCV screening results in 7769 avoided events of progression, which are associated with $€ 838.73$ million net savings accrued by the Italian NHS. The initial investment in treatment is recouped in 4.3 years in the form of savings from disease complications avoided. Conclusion Investment in treatment of newly diagnosed patients will bring a significant reduction in disease complications, which is associated with great economic benefits. This type of action can reduce the infection rate as well as the clinical and economic disease burden of HCV in Italy.
\end{abstract}

Andrea Marcellusi and Claudia Simonelli equally contributed and should be considered as joint first authors.

Loreta A. Kondili

loreta.kondili@iss.it

1 Economic Evaluation and HTA (EEHTA-CEIS),

Centre for Economic and International Studies, Faculty

of Economics, University of Rome "Tor Vergata”, Rome, Italy

2 Institute of Leadership and Management in Health, Kingston Business School, Kingston University, London, UK

3 Center for Global Health, Istituto Superiore di Sanità, Viale Regina Elena 299, 00161 Rome, Italy 


\section{Key Points for Decision Makers}

This analysis, based on real-life economic data from a representative sample of Italian $\mathrm{HCV}$-infected patients in care, confirms clinical and economic benefits associated with timely treatment of patients diagnosed through the approved screening programme in Italy.

As a result of the disease complications avoided, the Italian NHS can expect to break-even from the investment in anti-HCV treatment for patients diagnosed through screening in 4.3 years.

This evidence on cost benefits of treatment for patients is important for the ongoing decision-making process of Central and Regional decision makers, which are now responsible for the allocation of resources dedicated to Direct Acting Antiviral (DAA) treatment.

\section{Introduction}

Hepatitis $\mathrm{C}$ virus (HCV) is a leading cause of liver-related morbidity and mortality worldwide [1]. The development of direct-acting antiviral (DAA) therapy has revolutionised the approach to treatment, with real-life viral eradication of more than $98 \%$ among treated patients [2]. Considering the high real-life effectiveness of DAA, the World Health Organization (WHO) has set the goal of elimination of HCV infection by 2030 within the Global Health Sector Strategy on Viral Hepatitis 2016-2021 [3].

Italy has been considered the country with the highest $\mathrm{HCV}$ prevalence in the Western Europe, reported at around $1 \%$ [4]. Initially in Italy, HCV treatment with DAAs was prioritised to patients with severe, progressive liver disease. In 2017, a universal treatment strategy was introduced, meaning that all diagnosed patients were eligible for treatment [5]. Universal access to DAA therapies has resulted in higher annual treatments and is reported to generate stronger economic returns compared to prioritised access [6].

Considering that in Italy treatment was administered to the highest number of patients with chronic HCV infection in Europe, previous modelling analyses have named Italy among countries on track to achieve the WHO eliminations goals for $\mathrm{HCV}$, assuming that the number of treated patients remains high. Identifying these patients in order to maintain high annual treatment rates has become the true bottleneck for elimination in many on-track countries. Only the implementation of an active screening strategy could permit the identification of patients and their consequent treatment to eradicate the infection [7].

Indeed, there is a political will to achieve the elimination goals, as the need to identify undiagnosed individuals in the key high-risk groups and in the general population is recognised. In fact, based on evidence of the cost-effectiveness of a graduated screening strategy [7], in Italy a dedicated fund has recently been granted, which initially targets birth cohorts 1968-1988 and key populations [8]. Considering the state-of-the-art scenario, our work focused on evaluating the cost-consequences of treating $\mathrm{HCV}$-infected individuals diagnosed through active screening, implemented in Italy starting from 2020 . The final goal is to produce evidence that could support the extended HCV treatment investment in all infected individuals.

\section{Methods}

A previously validated Markov model [6] (Supplementary Material A1-Figure A1) was used to estimate clinical and economic outcomes of expanded access to DAA therapy of infected individuals who are estimated to be diagnosed through active screening in Italy, as approved by a recent Legislative Decree starting from the year 2020 [7, 8]. The number of disease complications avoided, and the costs saved from halting liver disease progression are estimated by the model over a 20 -year time horizon by comparing outcomes in treatment and no-treatment scenarios. A hypothetical cohort of 10,000 patients is entered into the model, stratified according to the fibrosis stage of people who will be diagnosed by screening. The analysis is from the perspective of Italian National Health System (NHS), and therefore only direct medical costs are considered.

The analysis is also performed for the treatment of diagnosed HCV-infected patients from the first use of DAA in 2015 until the year 2019, in order to compare outcomes between diagnosed and undiagnosed patients. The outcomes for this cohort have been re-estimated since this analysis is based on novel data on real-life costs of disease management. The impact of real-life costs on model outcomes for 2015-2019 were evaluated versus a recent modelling analysis that considers the same treatment period and costs from the literature [9].

\subsection{Model Structure}

A published Markov model [6] (Supplementary Material A1-Figure A1) capturing multiple states of morbidity and mortality was used to evaluate HCV disease progression and related costs for the treatment of 10,000 standardized patients over a 20 -year time horizon. The model structure considers 13 disease states (fibrosis stages from 
F0 to F4, decompensated cirrhosis (DC), hepatocellular carcinoma (HCC), liver transplant (LT) procedure, LT in following years, sustained virologic response (SVR) from F0 to F3, SVR from irreversible liver damage (ILD), HCVrelated death, and death from other causes) and 41 transition probabilities [6]. Events constituting advanced liver disease, such as ILD or DC, were considered as cumulative events in the model and not mutually exclusive [6]. In disease states DC, HCC, LT (procedure), or LT (following years) the event of death is assumed to be HCV-related [6].

Two different patient cohorts were simulated in the model to reflect two different time periods:

1. The first cohort represents patients who have received treatment between 2015 and 2019, according to the treatment eligibility criteria over time.

2. The second cohort represents patients diagnosed through the approved screening programme, treated immediately and independent of the fibrosis stage.

The model inputs, shown in Table 1 may differ according to which cohort is analysed in the model.

\subsection{Transition Probabilities}

In each annual cycle, patients could stay at their current liver disease stage or progress to a worse state according to the natural history of the disease. The probabilities of progressing through disease stages are obtained from the references considered in previous modelling [6, 10-13]. If patients receive treatment, natural progression can be slowed or stopped. The model assumes that when SVR is achieved, from stages F0-F3 liver damage is reversed, while from F4, DC, and HCC patients could incur additional liver damage (probability weighted for the percentage of patients with compensated cirrhosis, DC, and HCC) [6]. As in previous modelling, $10 \%$ of patients in $\mathrm{F} 3$ were assumed to have undiagnosed F4 liver fibrosis [9]. For the HCC state, the probability of death due to $\mathrm{HCV}$ and the probability of transplant were assumed to be independent [6]. Official national data on the competing probabilities of death from other causes was used to adjust the model transition probabilities $[9,16]$. Mortality has been adjusted depending on the cohort simulated, in order to account for the lower average age of the cohort treated post-screening (Italian health authorities have approved active screening of HCV starting from individuals that are $30-50$ years old).

\subsection{Treatment Efficacy}

The efficacy of DAAs was expressed in terms of the probability of reaching SVR (viral eradication by antiviral therapy) according to stage of fibrosis and genotype. The overall efficacy of second-generation, interferon-free HCV DAA regimens in 2015-2019 were obtained from the Italian Platform for the Study of Viral Hepatitis Therapies (PITER), a real-life representative cohort of $\mathrm{HCV}$-treated patients in Italy [14]. According to the number of patients treated in each year, data on efficacy were aggregated to obtain an overall treatment effectiveness for the cohort treated from 2015 to 2019. For the post-screening cohort, the effectiveness reported in 2019 was considered [2, 14].

\subsection{Epidemiological and Clinical Parameters}

Real-life data on the fibrosis stage and genotype distribution for HCV-infected patients treated in Italy from 2015 to 2019 were obtained from the Italian Drug Agency (AIFA) registry for DAA monitoring [5]. The distribution of fibrosis stage of patients who are expected to be diagnosed following active screening was estimated by a previous modelling conducted to evaluate the cost-effectiveness of active $\mathrm{HCV}$ screening in Italy $[7,15]$. The number of patients entered into the model are summarised in Supplementary Material A2.

\subsection{Economic Parameters}

The average treatment cost is based on non-official sources validated by an exert panel and also by authors of this article [9]. In 2015, 2016, and 2017 the price considered is $€ 25,000$, $€ 15,000$ and $€ 9000$, respectively [6]. From 2018, the treatment cost is reasonably assumed to be equal to $€ 6000$ per DAA treatment. According to the number of patients treated in each year, treatment prices were aggregated to obtain an overall treatment price for the cohort treated from 2015 to $2019(€ 11,801)$. For the post-screening cohort, the treatment price suggested for 2019 was considered (€6000).

Direct healthcare costs were those associated with the management of HCV-related diseases, including outpatient visits, biochemical analyses, and instrumental procedures. For the stages F0-F4, the direct costs of disease management were obtained for the first time in this study from the reallife PITER cohort [14]. Annual costs pre-SVR were assumed to remain constant in time. The associated per-patient cost by disease stage post-SVR was adapted to a decreasing trend in time based on real-life data on costs incurred in the first, second, and third year following SVR or SVR from ILD.

Full details on the elaboration of real-life costs based on the PITER cohort data are presented in Supplementary Material A3 (average frequency of resource use, unit costs of health services, resulting average costs of disease management per fibrosis stage before and after treatment response). In Supplementary Material A4 we have provided for additional information on the calculation of costs beyond three years. For the disease stages DC, HCC, LT (procedure), and LT (following years) the costs of disease management were 
Table 1 Model parameters

\begin{tabular}{|c|c|c|c|c|c|c|}
\hline & Base-case & Min & Max & Alpha & Beta & Source \\
\hline \multicolumn{7}{|l|}{ Probability of disease progression } \\
\hline \multicolumn{7}{|l|}{ (Annual) } \\
\hline $\mathrm{F} 0$ to $\mathrm{F} 1$ & 0.117 & 0.090 & 0.140 & 119.73 & 903.57 & {$[10]$} \\
\hline $\mathrm{F} 1$ to $\mathrm{F} 2$ & 0.085 & 0.070 & 0.102 & 124.10 & 1335.91 & {$[10]$} \\
\hline $\mathrm{F} 2$ to $\mathrm{F} 3$ & 0.120 & 0.100 & 0.144 & 119.32 & 874.98 & {$[10]$} \\
\hline $\mathrm{F} 3$ to $\mathrm{F} 4$ & 0.100 & 0.080 & 0.120 & 122.05 & 1098.45 & [11] \\
\hline F4 to DC & 0.030 & 0.020 & 0.036 & 131.62 & 4255.74 & {$[11]$} \\
\hline $\mathrm{F} 4$ to $\mathrm{HCC}$ & 0.050 & 0.040 & 0.060 & 128.89 & 2448.84 & {$[11]$} \\
\hline $\mathrm{DC}$ to $\mathrm{HCC}$ & 0.100 & 0.080 & 0.120 & 122.05 & 1098.45 & [11] \\
\hline $\mathrm{DC}$ to $\mathrm{TP}$ & 0.110 & 0.090 & 0.132 & 120.68 & 976.44 & {$[11]$} \\
\hline $\mathrm{HCC}$ to TP & 0.200 & 0.160 & 0.240 & 108.38 & 433.51 & {$[12]$} \\
\hline SVR to HCC & 0.006 & 0.007 & 0.009 & 25.77 & 4147.81 & [13] \\
\hline SVR to TP & 0.009 & 0.011 & 0.020 & 4.13 & 437.71 & [13] \\
\hline \multicolumn{7}{|l|}{ Probability of progressing to death } \\
\hline \multicolumn{7}{|l|}{ (Annual) } \\
\hline DC to death (1.r.) & 0.090 & 0.070 & 0.108 & 123.42 & 1247.89 & {$[12]$} \\
\hline HCC to death (1.r.) & 0.430 & 0.340 & 0.516 & 76.93 & 101.98 & [13] \\
\hline TP (procedure) to death (1.r.) & 0.150 & 0.120 & 0.180 & 115.21 & 652.88 & [13] \\
\hline TP (following years) to death (1.r.) & 0.057 & 0.050 & 0.068 & 127.93 & 2116.44 & [13] \\
\hline Mortality (2015-2019) & 0.062 & 0.043 & 0.103 & 11.31 & 17.38 & [9] \\
\hline Mortality (post-screening) & 0.044 & 0.039 & 0.069 & 40.54 & 885.98 & [9] \\
\hline \multicolumn{7}{|l|}{ DAA effectiveness } \\
\hline \multicolumn{7}{|l|}{ 2015-2019 } \\
\hline F0-F3 to SVR (G1) & 0.969 & 0.793 & 1.000 & 161.06 & 5.23 & {$[14]$} \\
\hline F4-DC to SVR (G1) & 0.950 & 0.760 & 1.000 & 96.08 & 5.10 & {$[14]$} \\
\hline F0-F3 to SVR (G2) & 0.928 & 0.767 & 1.000 & 64.13 & 4.97 & {$[14]$} \\
\hline F4-DC to SVR (G2) & 0.917 & 0.671 & 1.000 & 54.06 & 4.90 & [14] \\
\hline F0-F3 to SVR (G3) & 0.895 & 0.730 & 1.000 & 40.64 & 4.76 & {$[14]$} \\
\hline F4-DC to SVR (G3) & 0.882 & 0.705 & 1.000 & 35.01 & 4.67 & {$[14]$} \\
\hline F0-F3 to SVR (G4+) & 0.831 & 0.690 & 1.000 & 21.28 & 4.34 & [14] \\
\hline F4-DC to SVR (G4+) & 0.801 & 0.667 & 1.000 & 16.66 & 4.15 & {$[14]$} \\
\hline \multicolumn{7}{|l|}{ Post-screening } \\
\hline F0-F3 to SVR (G1) & 0.980 & 0.784 & 1.000 & 259.72 & 5.30 & {$[2,14]$} \\
\hline F4-DC to SVR (G1) & 0.980 & 0.745 & 1.000 & 259.72 & 5.30 & {$[2,14]$} \\
\hline F0-F3 to SVR (G2) & 0.980 & 0.784 & 1.000 & 259.72 & 5.30 & {$[2,14]$} \\
\hline F4-DC to SVR (G2) & 0.980 & 0.776 & 1.000 & 259.72 & 5.30 & {$[2,14]$} \\
\hline F0-F3 to SVR (G3) & 0.980 & 0.760 & 1.000 & 259.72 & 5.30 & {$[2,14]$} \\
\hline F4-DC to SVR (G3) & 0.980 & 0.707 & 1.000 & 259.72 & 5.30 & {$[2,14]$} \\
\hline F0-F3 to SVR (G4+) & 0.980 & 0.776 & 1.000 & 259.72 & 5.30 & {$[2,14]$} \\
\hline F4-DC to SVR (G4+) & 0.980 & 0.769 & 1.000 & 259.72 & 5.30 & {$[2,14]$} \\
\hline \multicolumn{7}{|l|}{ Fibrosis distribution } \\
\hline \multicolumn{7}{|l|}{ 2015-2019 } \\
\hline$\% \mathrm{~F} 0$ & 0.098 & 0.078 & 0.117 & 122.39 & 1132.49 & {$[5]$} \\
\hline$\% \mathrm{~F} 1$ & 0.098 & 0.078 & 0.117 & 122.39 & 1132.49 & {$[5]$} \\
\hline$\% \mathrm{~F} 2$ & 0.183 & 0.147 & 0.220 & 110.69 & 493.99 & {$[5]$} \\
\hline$\%$ F3 & 0.208 & 0.166 & 0.249 & 107.32 & 409.36 & {$[5]$} \\
\hline$\%$ F4-cirrhosis & 0.347 & 0.451 & 0.244 & 39.33 & 73.85 & {$[5]$} \\
\hline$\% \mathrm{DC}$ & 0.044 & 0.053 & 0.035 & 129.66 & 2793.77 & {$[5]$} \\
\hline$\% \mathrm{HCC}$ & 0.022 & 0.027 & 0.018 & 132.67 & 5806.72 & [5] \\
\hline
\end{tabular}


Table 1 (continued)

\begin{tabular}{|c|c|c|c|c|c|c|}
\hline & Base-case & Min & Max & Alpha & Beta & Source \\
\hline$\% \mathrm{~F} 0$ & 0.160 & 0.171 & 0.149 & 218.82 & 1040.08 & {$[7,15]$} \\
\hline$\% \mathrm{~F} 1$ & 0.226 & 0.242 & 0.210 & 199.99 & 617.74 & {$[7,15]$} \\
\hline$\% \mathrm{~F} 2$ & 0.147 & 0.158 & 0.137 & 222.52 & 1169.21 & {$[7,15]$} \\
\hline$\% \mathrm{~F} 3$ & 0.205 & 0.219 & 0.190 & 206.07 & 723.31 & {$[7,15]$} \\
\hline$\%$ F4-cirrhosis & 0.214 & 0.171 & 0.257 & 13.70 & 70.24 & {$[7,15]$} \\
\hline$\% \mathrm{DC}$ & 0.027 & 0.022 & 0.033 & 16.20 & 762.69 & {$[7,15]$} \\
\hline$\% \mathrm{HCC}$ & 0.021 & 0.017 & 0.025 & 16.29 & 1001.55 & {$[7,15]$} \\
\hline \multicolumn{7}{|l|}{ Genotype distribution } \\
\hline$\% \mathrm{G} 1$ & 0.608 & 0.730 & 0.487 & 52.54 & 33.82 & [5] \\
\hline$\% \mathrm{G} 2$ & 0.179 & 0.215 & 0.143 & 111.22 & 509.44 & [5] \\
\hline$\% \mathrm{G} 3$ & 0.137 & 0.035 & 0.238 & 8.39 & 52.87 & [5] \\
\hline$\% \mathrm{G} 4$ & 0.076 & 0.020 & 0.132 & 9.05 & 110.78 & [5] \\
\hline \multicolumn{7}{|l|}{ Treatment price } \\
\hline Treatment price (2015-2019) & $€ 11,801$ & $€ 9441$ & $€ 13,781$ & 192.92 & 61.17 & [6], L.E. opinion \\
\hline Treatment price (post-screening) & $€ 6000$ & $€ 4800$ & $€ 7200$ & 135.72 & 44.21 & [6], L.E. opinion \\
\hline \multicolumn{7}{|l|}{ Other direct medical expenses } \\
\hline F0 & $€ 111.02$ & $€ 100.79$ & $€ 121.26$ & 638.37 & 0.17 & [14] \\
\hline F1 & $€ 111.02$ & $€ 100.79$ & $€ 121.26$ & 638.37 & 0.17 & [14] \\
\hline $\mathrm{F} 2$ & $€ 114.13$ & $€ 98.46$ & $€ 129.80$ & 287.95 & 0.40 & [14] \\
\hline F3 & $€ 116.41$ & $€ 94.59$ & $€ 138.22$ & 154.65 & 0.75 & {$[14]$} \\
\hline F4-cirrhosis & $€ 474.42$ & $€ 268,68$ & $€ 680.16$ & 28.87 & 16.43 & {$[14]$} \\
\hline SVR from F0-F3 ( $1^{\circ}$ year) & $€ 93.61$ & $€ 72.94$ & $€ 114.33$ & - & - & [14] \\
\hline SVR from F0-F3 ( $2^{\circ}$ year) & $€ 69.49$ & $€ 39.81$ & $€ 99.24$ & - & - & {$[14]$} \\
\hline SVR from F0-F3 ( $3^{\circ}$ year) & $€ 63.27$ & $€ 5.79$ & $€ 139.82$ & - & - & {$[14]$} \\
\hline SVR from F4-DC ( $1^{\circ}$ year $)$ & $€ 1030.11$ & $€ 633.66$ & $€ 1426.56$ & - & - & {$[14]$} \\
\hline SVR from F4-DC (2 year $)$ & $€ 928.85$ & $€ 545.73$ & $€ 1311.97$ & - & - & {$[14]$} \\
\hline SVR from F4-DC ( $3^{\circ}$ year) & $€ 921.23$ & $€ 496.34$ & $€ 1436.65$ & - & - & {$[14]$} \\
\hline \multicolumn{7}{|l|}{ Other direct medical expenses } \\
\hline DC & $€ 6626.50$ & $€ 4385.00$ & $€ 8868.00$ & 47.45 & 139.66 & {$[6]$} \\
\hline $\mathrm{HCC}$ & $€ 12,896.00$ & $€ 5792.00$ & $€ 20,000.00$ & 17.89 & 720.84 & [6] \\
\hline $\mathrm{TP}\left(1^{\circ}\right.$ year $)$ & $€ 73,774.00$ & $€ 62,648.00$ & $€ 84,900.00$ & 238.69 & 309.07 & [6] \\
\hline TP (following years) & $€ 2364.50$ & $€ 0.00$ & $€ 4729.00$ & 5.43 & 435.54 & [6] \\
\hline
\end{tabular}

$C I$ confidence interval, $D A A$ direct-acting antiviral, $D C$ decompensated cirrhosis, $F 0-F 4$ fibrosis stages, $G 0-G 4+$ genotypes, $H C C$ hepatocellular carcinoma, L.E. local expert, l.r. liver-related, S.M. supplementary material, $S V R$ sustained virologic response, $T P$ transplant

obtained from literature data and the costs post-SVR were reasonably assumed to remain constant in time [6].

\subsection{Scenarios}

The model simulates the progression of 10,000 standardised HCV-infected patients over a 20-year time horizon according to two different treatment scenarios:

1. No-treatment scenario: patients in disease stages F0-F4 follow the natural history of $\mathrm{HCV}$ without any therapy.

2. Treatment scenario: patients in disease stages F0-F4 receive treatment for $\mathrm{HCV}$.
Each of these scenarios is run for the patient cohorts reported above (Sect. 2.1), to reflect the two different time periods of treatment (i.e., years 2015-2019 treated patient cohort and years following 2020, defined as the post-screening cohort).

\subsection{Economic Analysis}

The economic analysis was performed by comparing the cumulative cost of treating 10,000 standardised patients with the costs sustained given the natural history of the disease with no antiviral therapy. The cost difference between treatment and no-treatment scenarios represents the savings that could be accrued by the NHS as a consequence of viral 
elimination, net of the initial investment in treatment. The break-even point in time (BPT) was calculated, i.e., the time (in years) required for the initial investment in DAAs to be recovered in terms of cost savings from treatment. The economic analysis was performed for both cohorts (2015-2019 cohort and post-screening cohort). Costs were expressed in Euros at the 2019 price level and were discounted at an annual rate of $3 \%$.

\subsection{Sensitivity Analysis}

The uncertainty associated with the model outcomes was estimated through a deterministic sensitivity analysis (DSA) and a probabilistic sensitivity analysis (PSA). The DSA explains the variability of the results associated with specific parameters in isolation, while in the PSA inputs vary simultaneously to obtain the overall uncertainty around model outcomes. The probabilistic distribution choice for the PSA was made by applying a gamma distribution for economic parameters and a beta distribution for epidemiological parameters [17].

The parameters tested are annual probabilities of disease progression, annual probabilities of progressing to death, probability of death from all other causes, treatment efficacy, fibrosis and genotype distribution, treatment price, other direct medical costs, and discount rate. The minimum and maximum values for each parameter were obtained by considering an arbitrary constant variation $( \pm 20 \%)$ with respect to the base-case value. For direct costs of disease management obtained from the real-life data, the bounds of the $95 \%$ confidence intervals (CI) were considered. The minimum values for treatment effectiveness are obtained from previous modelling [6], while the maximum values are set equal to 1 . DSA ranges ( $\mathrm{min} / \mathrm{max}$ values simulated) and PSA parameters are reported in Table 1.

The variability of costs post-SVR, as detailed in Supplementary Material A4, is defined in the PSA by applying a normal distribution to the coefficients of the logarithmic equation estimated for decreasing costs (Supplementary Material A4-Table A4.2). For transition probabilities, given the large variability reported the literature, in the DSA the values tested are obtained from Linthicum et al. (2016) [18] (Supplementary Material A5-Table A5.1).

The results obtained in the DSA are represented by tornado diagrams. For the PSA, 5000 Monte Carlo simulations were performed in order to obtain $95 \%$ CI for disease complications avoided, cost reductions at 20 years, and BPT. The Monte Carlo simulations were also used to plot, for both scenarios, the probability of reaching break-even for each year after the patients are entered in the model. Both sensitivity analyses have been undertaken for the 2015-2019 and for the post-screening cohorts.

\section{Results}

The model outcomes are summarised in Table 2. By expanding access to DAA therapies in newly diagnosed patients through an active screening, there would be 7769 fewer events over the next 20 years (95\% CI 5386-8819) (Table 2). Expanding treatment in infected but not yet diagnosed individuals would save $€ 838.73$ million (CI 95\% 572.82-1,163.71) for 10,000 treated patients in Italy. It would take 4.3 years (95\% CI 3.80-6.05) to reach the BPT, thus the initial investment on antiviral treatment could be recovered in approximately in 4.3 years (Table 2 ).

Considering the real-life costs of disease management, for 10,000 patients treated in 2015-2019 there are 8859 avoided events of progression over the model horizon $(95 \%$ CI 6498-11,927). The events of progression avoided from treating diagnosed patients, first in a prioritised manner and then expanding to universal access in 2017, has resulted in savings equal to $€ 654.5$ million for 10,000 patients $(95 \% \mathrm{CI}$ 317.21-1154.73). The initial investment in antiviral treatment is estimated to be recovered in approximately 5.5 years (95\% CI 3.75-6.08).

Figure 1 provides the graphic representation of cumulative disease complications avoided and cumulative costsavings throughout the model horizon for both scenarios versus the respective no-treatment scenario. In each year, the disease complications avoided in the treatment scenario due to viral eradication by antiviral treatment (represented by the bars in Fig. 1) are lower in the post-screening scenario compared to the 2015-2019 scenario. The curve in Fig. 1 shows the additional cumulative expenditure that the NHS would incur for treatment in comparison to the respective no-treatment scenario. In the first year, the additional expenditure is represented by the investment in treatment, and the curve decreases thereafter as a result of savings from avoided disease complications.
Table 2 Avoided disease complications and savings over 20 years and BPT

\begin{tabular}{llll}
\hline Results & $\begin{array}{l}\text { Avoided disease complications } \\
\text { after 20 years }(95 \% \mathrm{CI})\end{array}$ & $\begin{array}{l}\text { Savings after 20 years, € million } \\
(95 \% \mathrm{CI})\end{array}$ & BPT years (95\% CI) \\
\hline Post-screening & $7769(5386-8819)$ & $€ 838.73(€ 572.82-€ 1163.71)$ & $4.3(3.80-6.05)$ \\
$2015-2019$ & $8859(6498-11,927)$ & $€ 654.50(€ 317.21-€ 1154.73)$ & $5.5(3.75-6.08)$ \\
\hline
\end{tabular}

$B P T$ break-even point in time, $C I$ confidence interval 


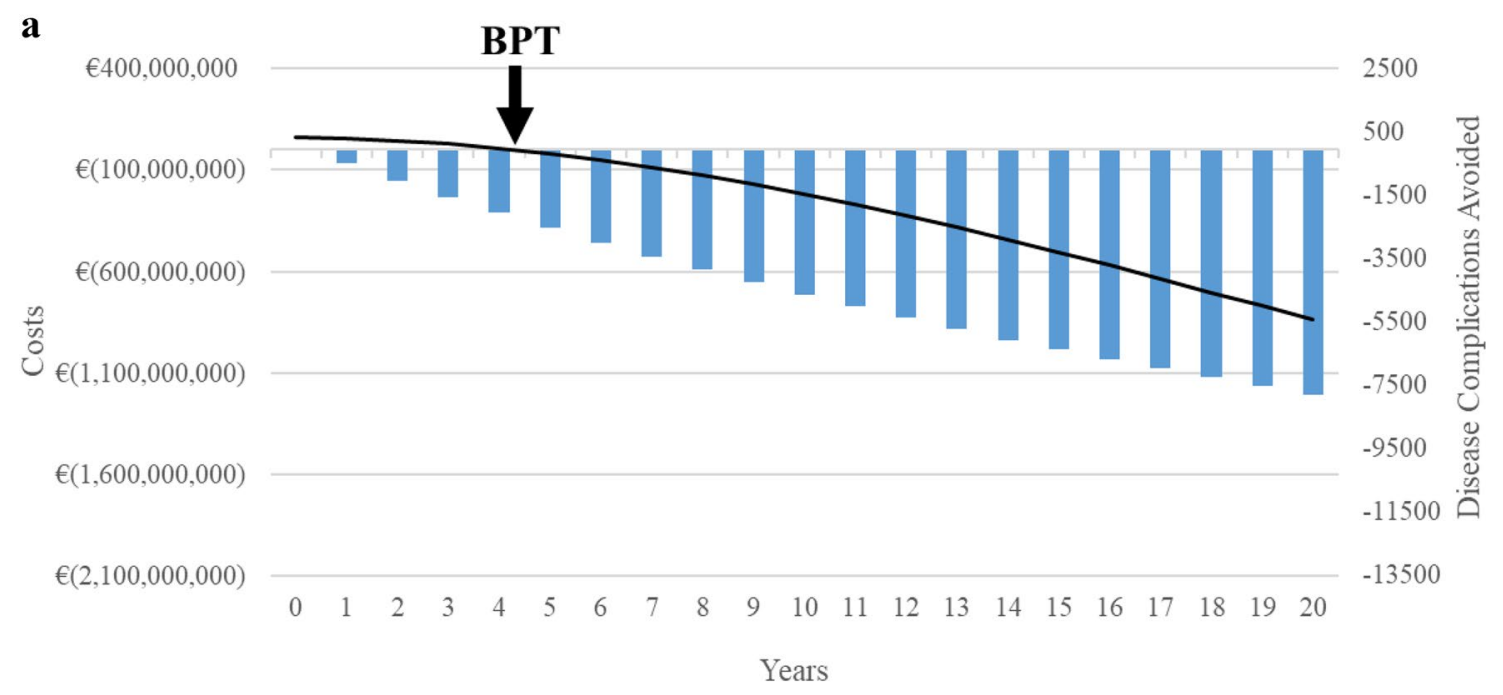

b

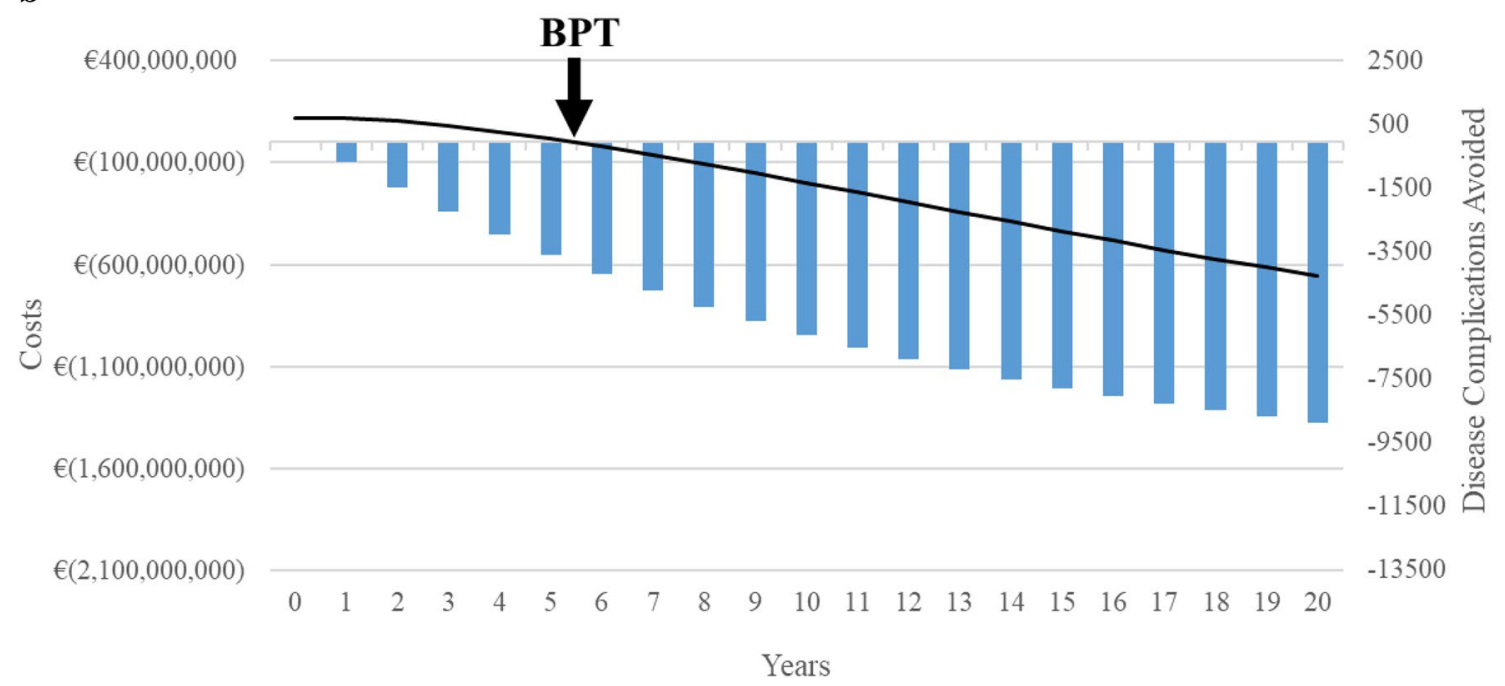

Fig. 1 Comparison of clinical and economic impact of DAA treatment according to different treatment policies. a Disease complications avoided and cost-savings (post-screening) over 20 years and

The tornado diagrams in Fig. 2 illustrate the results of the DSA. Considering the transition probabilities reported by Linthicum et al. (2016) [18], the BPT increases by 53\% in 2015-2019 and by 44\% in the post-screening scenario. These parameters generate most uncertainty in the BPT and also strongly impact potential savings ( $-41 \%$ in 2015-2019 and $-31 \%$ in post-screening). The costs of disease management also have a considerable impact on results: the minimum values considered for DSA generate lower cost-savings (-34\% in post-screening - 38\% in 2015-2019) and a higher BPT $(+19 \%$ in post-screening and $+23 \%$ in $2015-2019)$. Similarly, the maximum values of disease management costs generate higher cost-savings $(+18 \%$ in post-screening and $+22 \%$ in $2015-2019)$ and a lower BPT (- $9 \%$ in postscreening and $-11 \%$ in $2015-2019)$. Finally, cost-savings
BPT. b Disease complications avoided and cost-savings (2015-2019) over 20 years and BPT. DAA direct-acting antiviral, BPT break-even point in time

are very sensitive to the probability of death from other causes, which generate a variation ranging from -46 to $32 \%$ in $2015-2019$ and from -20 to $8 \%$ in post-screening.

Treatment effectiveness, treatment prices and fibrosis distribution also affect model outputs, although more moderately with respect to transition probabilities, medical costs, and mortality rates. In spite of the lower returns that could result from potentially higher lower/higher values of certain parameters in real-life, for both scenarios in the sensitivity analysis cost-savings are never equal to zero and the BPT is never beyond the model time horizon.

The 95\% CIs obtained with the PSA are reported in Table 2. For all model outputs (disease complications avoided, cost-savings, and BPT) the CI around basecase results are wider in the 2015-2019 scenario than 
$\mathbf{a}$

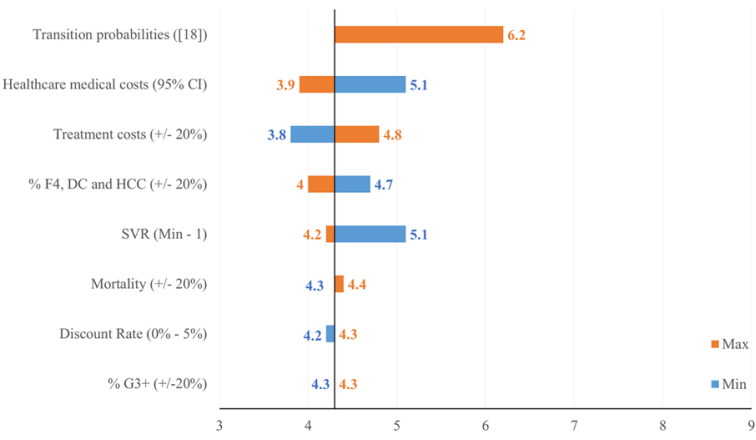

b

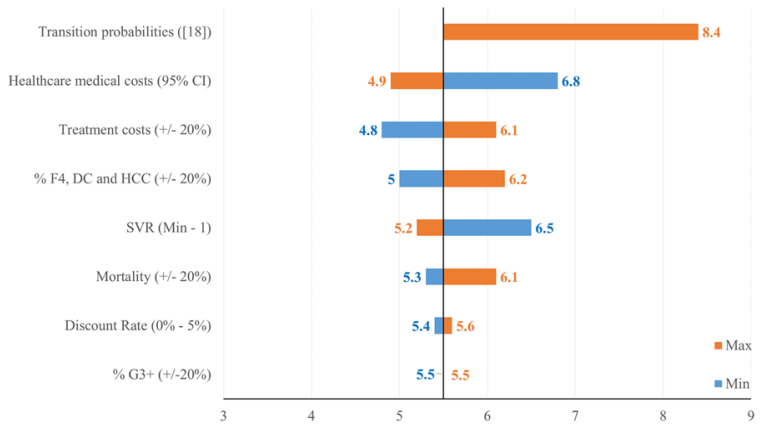

Fig. 2 Deterministic sensitivity analysis (DSA)—tornado diagrams. a DSA: cost-savings, €million (post-screening); b DSA: BPT (postscreening) c DSA: cost-savings, €million (2015-2019); d DSA: BPT

in post-screening. The larger variability in the first scenario also emerged in the DSA analysis (Fig. 2), where the parameters with most impact generate a larger percentage variation in 2015-2019 as opposed to post-screening. Still, in both scenarios the CI for cost-savings do not contain the value zero, and therefore it is confirmed that treatment intervention is a significant cost-saving strategy.

Figure 3 illustrates the probability of achieving the BPT in each year of follow-up for both scenarios. After five years, for instance, the probability of break-even from the initial investment is equal to $65.5 \%$ for patients diagnosed and treated following an active screening and is equal to $21.0 \%$ for patients who have been treated in 2015-2019. In general, in each given year of follow-up, the probability of breaking even in the post-screening scenario is higher than the 2015-2019 scenario. The Italian NHS would almost certainly break-even from the initial investment within 8 years in both scenarios.

\section{Discussion}

In order to reach WHO elimination goals by the year 2030, Italy should maintain the number of treated patients as high as 40,000/year [19]. However, without active screening c

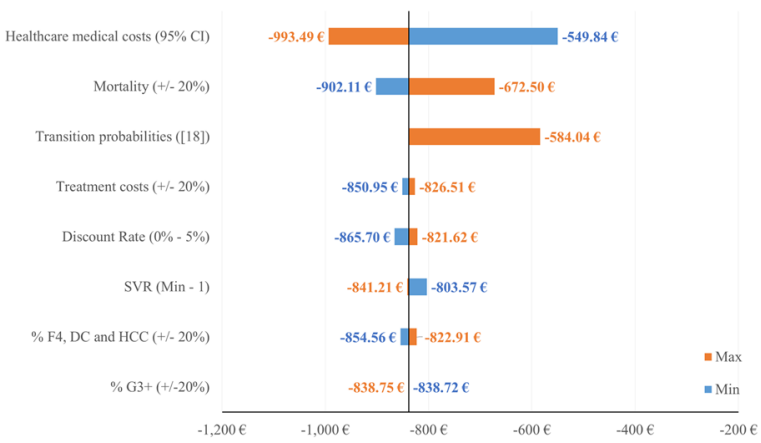

d

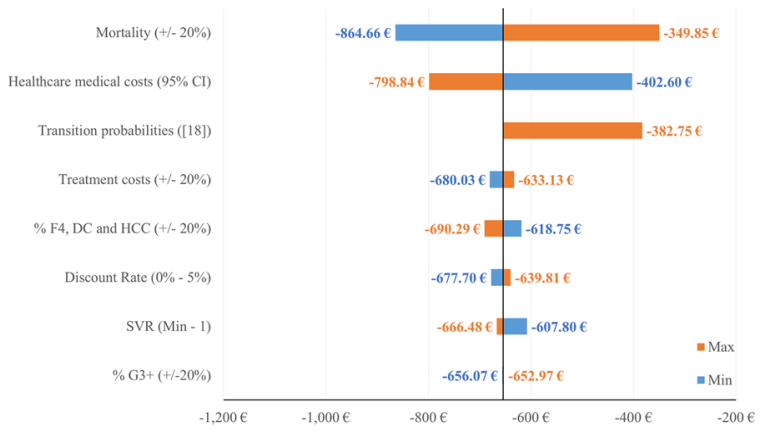

(2015-2019). BPT break-even point in time, $C I$ confidence interval, $D C$ decompensated cirrhosis, G3+ all genotypes except G1 and G2, $H C C$ hepatocellular carcinoma, $S V R$ sustained virologic response

strategies, the number of patients eligible for treatment (diagnosed patients with chronic HCV infection) is estimated to run out within the years 2023-2025 and around 300,000 HCV-infected individuals would remain undiagnosed [7, 15]. Considering the reported data, and that already in 2019 treatment rates have started to decline, Italy was removed from countries that are on-track for HCV elimination [20].

In addition to being a fundamental policy aspect for HCV elimination, screening is also reported to be cost effective in Italy [21]. For this reason, in 2020, a fund for HCV screening was allocated [8]. However, it is crucial to define health policies that not only identify undiagnosed infections but also guarantee timely access to treatment for all infected individuals [22]. The results of our evaluation indicate significant cost savings from immediate treatment of the patients diagnosed through active screening. Across a 20 -year time horizon, for 10,000 patients there are $€ 838.73$ million in cost-savings associated with administration of antiviral treatment. In addition, the Italian NHS can expect to break-even from the initial investment in DAAs for all patients diagnosed within 4.3 years. 
Fig. 3 Probability of reaching the BPT per year. BPT breakeven point in time

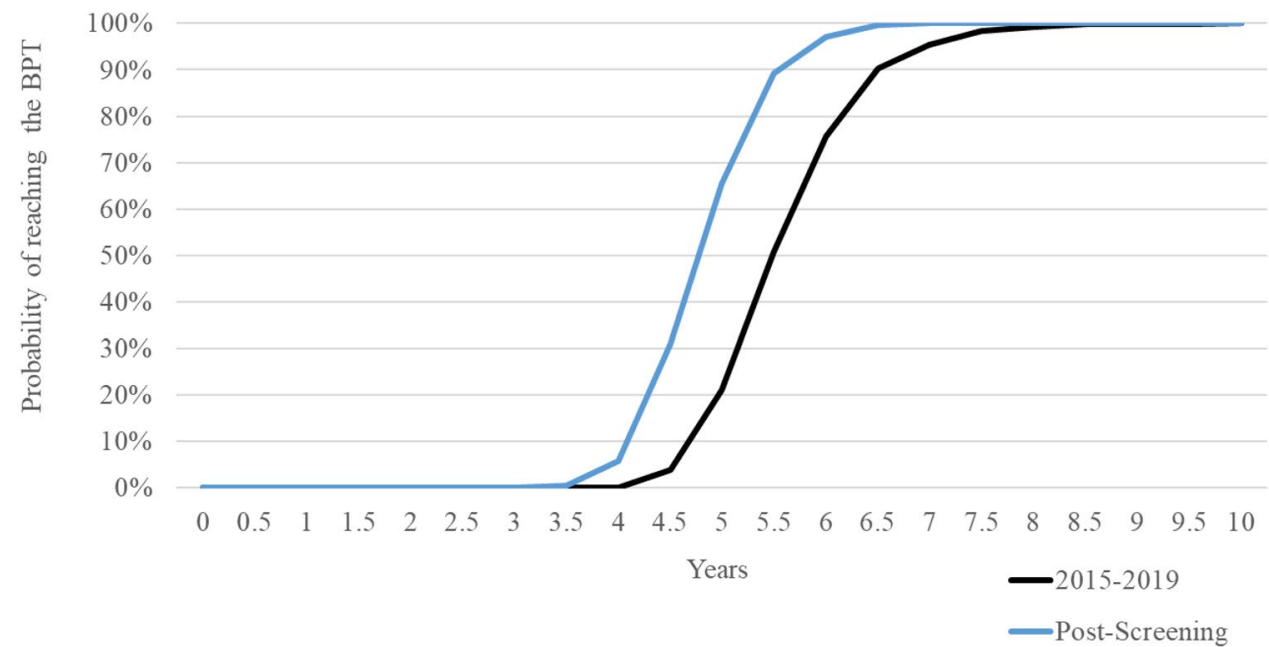

In spite of the different variabilities of inputs that compose the calculation of cost-savings and BPT, in the worst hypothesis, according to the results of the PSA, the return of investments is expected 8 years after successful treatment. In fact, as reported by our data, it is possible to see that at the 8 th year the probability of achieving BPT is close to $100 \%$ (Fig. 3). Starting from 2021, if treatment were to be administered universally and without delays to the patient population diagnosed through active screening, then the Italian NHS would almost certainly start to accrue savings from the initial investment in treatment before 2030 .

In this analysis, we have additionally estimated data on the cost-consequences of treating patients in 2015-2019 in order to discuss how model outcomes change when we consider the population that has been treated and the population that will be treated. With respect to existing literature, the outcomes for patients treated in 2015-2019 are based on real-life data on the costs of disease management by fibrosis stage obtained from the PITER cohort (Supplementary Material A3). These costs can be considered representative of the population of treated patients, since individuals enrolled in PITER are a representative cohort of patients in care in Italy [23].

When considering real-life costs of HCV disease management for different liver disease stages, it is confirmed that treatment throughout the overall period of their use (2015-2019) generates important cost savings (€654.50 million for 10,000 standardised patients). In an existing evaluation on the same treatment phase, the savings reported are equal to $€ 63.50$ million for 1000 patients simulated [9]. This difference should depend on the different disease management costs per fibrosis stage considered (literature empirical costs vs costs from the real-life PITER cohort used in the present study). In PITER, lower annual costs per fibrosis stage emerge, which should result in lower cost savings. However, we have also estimated a lower residual cost of disease management after response to treatment and a decreasing (rather than constant) trend of annual costs of disease management after viral eradication, which may explain the higher estimates in this analysis with respect to previous modelling on the same period.

With respect to 2015-2019, in the post-screening scenario there are lower disease complications avoided (7769 complications in post-screening vs 8859 complications in 2015-2019), despite the improved treatment effectiveness. This is due to the higher proportion of patients with endstage liver disease reported during the years 2015-2019 with respect to infected non-diagnosed individuals (advanced liver disease of fibrosis stage F4 are $41.4 \%$ in $2015-2019$ vs $26.2 \%$ in post-screening). For a given interval of time, patients in advanced stages of disease progression have more frequent complications. In previous literature, an inverse correlation between the number of complications avoided and cost-savings is reported. In fact, the non-progression of patients with liver fibrosis stages lower than F3 generates important cost reductions over time because no residual cost time remains after viral eradication [6]. In our analysis, we observe a similar outcome: for 10,000 patients, costs saved after 20 years are equal to $€ 838.73$ million in post-screening and $€ 654.50$ million in 2015-2019. In addition, the NHS can expect to break-even from the initial investment in less time (5.5 years in 2015-2019 vs 4.4 years in post-screening).

If we consider the parameters that change across cohorts (mortality rate, fibrosis distribution, treatment effectiveness and treatment costs) and the results of the DSA, it is possible to make several considerations. First, the price renegotiations are a key reason why the NHS can expect to breakeven earlier from initial investment in treatment. Also, in the post-screening scenario we have considered, as suggested by literature [21], a cohort with a lower average age with respect to the years 2015-2019. We have incorporated this difference by introducing a lower probability of dying from 
all other causes in post-screening. As shown in Fig. 2, a lower mortality rate has a strong, positive impact on costsavings, and could therefore be a driver of better economic outcomes for patients yet to be diagnosed. This is reasonable, since patients who are younger on average are less likely to die from other causes, and by remaining alive these would present worsening health conditions that are avoided by treatment. Small improvements in treatment effectiveness and the difference in fibrosis distribution actually have moderate effects on model outcomes.

Our data are supportive of a health policy which not only guarantees diagnosis and screening, but also investment for immediate $\mathrm{HCV}$ treatment of diagnosed patients in order to prevent the continuous progression of liver disease over time and potential development of advanced liver damage, which bring severe clinical and economic consequences. This evidence on cost benefits of treatment for patients is important for the ongoing decision-making process of Central and Regional decision makers, who are responsible for the allocation of resources dedicated to DAA treatment of all newly diagnosed HCV infected individuals. We feel that establishing an $a d$ hoc fund for DAAs for each region binding resources both for case finding through active screening and treatment within the National Plan for the Prevention and Treatment of Hepatitis $\mathrm{C}$ is of paramount importance, in order to keep Italy on track to achieve the WHO elimination targets by 2030 .

\subsection{Study Limitations}

Limitations to this modelling study exist. First, in existing literature there is an important variability in the reported probabilities of progressing across disease stages of liver disease. The uncertainty associated with transition probabilities represents a limitation for most research that models HCV disease progression. There are many aspects that create heterogeneity in the reported probabilities of disease progression, as their non-linearity, the host and environmental factors that may affect it, and their dependence (in the model) on the fibrosis stage, which may bias the outcomes. The fibrosis stage distribution is indeed based on modelling of the epidemiology of HCV in Italy, which is validated, updated and calibrated according to the data of disease register in Italy [7, 14], but the distribution is anyway associated with some uncertainty per se. These limitations are addressed by analysing the uncertainty around the model outcomes caused by these specific parameters.

Second, even though this analysis is mostly based on real-life, in some cases data were not available, and therefore obtained from the literature. This is the case for transition probabilities, for the medical expenses in some disease states (DC, HCC, and liver transplant in the first and following years), and for the fibrosis distribution of the currently undiagnosed population. Methodologically, the mixture of data from the literature and real-life is not fully appropriate; however, this approach considers all available evidence and represents a proxy of what decision-makers could expect from their public health decisions, especially considering the limited data available from the literature. Still, all parameters were subject to the sensitivity analysis.

Third, the reinfection possibility and its treatment were not considered in this analysis due to the lack of data in general population and considering its very low probability and impact in a general population. In a specific analysis for high-risk populations, this event should be considered in terms of cost benefit of treatment and time to return of treatment investment.

Fourth, real-life costs of disease management generate considerable uncertainty around model outcomes. This depends on the the variability of annual costs for disease management that have emerged from our analysis, and also the adaptation of costs post-SVR to a trend based on the initial three years of data. In fact, in some simulations of the sensitivity analysis, the uncertainty around costs post-SVR generate increasing trends of annual costs for disease management. This outcome was not excluded as there may be a chance that patients incur some permanent liver damage that may cause annual medical expenses to increase.

\section{Conclusion}

The results of this study indicate that the investment in DAA treatment of newly diagnosed patients by an active screening will bring a significant reduction in clinical events and generate great economic benefits. This action is a key element to achieve the WHO elimination goals for $\mathrm{HCV}$ infection, as it can guarantee the reduction of the infection rate and of the clinical and economic disease burden of chronic HCV infection in Italy.

Supplementary Information The online version contains supplementary material available at https://doi.org/10.1007/s40258-021-00677-x.

\section{Declarations}

Funding This study was funded by Italian Ministry of Health, Grant number RF-2016-02364053.

Conflict of interest AM, CS, FSM and LAK have no conflicts of interest to declare regarding the content of this article.

Compliance with ethical standards The PITER cohort study protocol was approved by the Ethical Committee of Istituto Superiore di Sanità and by the local ethics committees of each clinical centre. Patient data were evaluated via an anonymous analysis, adopting codes generated by the electronic case-report form. Informed consent was obtained from each patient participating in this study. 
Data availability statement Cost data were collected in the real life PITER cohort. The distribution of liver fibrosis in patients that will be diagnosed by treatment was based also in a modelling study [9] in which real life data of the PITER cohort were used. PITER is a multicentric prospective study that was approved by the Ethical Committee of Istituto Superiore di Sanità (Italian National Institute of Public Health) and the local ethical committees of each of clinical centres involved in the study. By protocol, the data are the property of the participating clinical centres, while Istituto Superiore di Sanità acts as coordinating centre for data management and analysis. Cumulative data are reported within the paper, whereas each patient's data are not fully available and without restrictions for ethical reasons. The datasets generated and analysed during the current study could be available upon request to all interested researchers.

Code availability The model was shared for the peer review process. Authors can give more details upon request.

Author contributions AM, FSM, LAK and CS designed the study. AM built the modelling and validated it. CS prepared the material, managed and analyzed the real-life cost of disease management, conducted the analysis and finalised the draft of the manuscript. AM, LAK and FSM provided guidance on the methodology, reviewed the results and critically assessed the manuscript. All of the authors reviewed the results of the final draft of the manuscript and approved the final version of the manuscript. LAK had full access to all the data used in the study and had final responsibility for the decision to submit for publication.

Open Access This article is licensed under a Creative Commons Attribution-NonCommercial 4.0 International License, which permits any non-commercial use, sharing, adaptation, distribution and reproduction in any medium or format, as long as you give appropriate credit to the original author(s) and the source, provide a link to the Creative Commons licence, and indicate if changes were made. The images or other third party material in this article are included in the article's Creative Commons licence, unless indicated otherwise in a credit line to the material. If material is not included in the article's Creative Commons licence and your intended use is not permitted by statutory regulation or exceeds the permitted use, you will need to obtain permission directly from the copyright holder. To view a copy of this licence, visit http://creativecommons.org/licenses/by-nc/4.0/.

\section{References}

1. World Health Organization. Global hepatitis report, 2017. Global hepatitis programme. 2017. https://www.who.int/publications/i/ item/global-hepatitis-report-2017. Accessed 12 Dec 2020.

2. European Association for the Study of the Liver. EASL recommendations on treatment of hepatitis C: final update of the series. European Association for the Study of the Liver. J Hepatol. 2020;73(5):1170-218.

3. World Health Organization. Global health sector strategy on viral hepatitis 2016-2021. Glob. Hepat. Program. Dep. HIV/ AIDS. 2016. https://apps.who.int/iris/bitstream/handle/10665/ 246177/WHO-HIV-2016.06-eng.pdf? sequence $=1 \&$ isAllowed $=\mathrm{y}$. Accessed 12 Dec 2020.

4. Andriulli A, et al. Declining prevalence and increasing awareness of HCV infection in Italy: a population-based survey in five metropolitan areas. Eur J Intern Med. 2018;53:79-84. https://doi. org/10.1016/j.ejim.2018.02.015.

5. "Aggiornamento epatite ClAgenzia Italiana del Farmaco. https:// www.aifa.gov.it/aggiornamento-epatite-c. Accessed 22 Dec 2020.
6. Marcellusi A, et al. Economic consequences of investing in anti$\mathrm{HCV}$ antiviral treatment from the Italian NHS perspective: a real-world-based analysis of PITER data. Pharmacoeconomics. 2019;37(2):255-66. https://doi.org/10.1007/s40273-018-0733-3.

7. Kondili LA, et al. Forecasting Hepatitis $\mathrm{C}$ liver disease burden on real-life data. Does the hidden iceberg matter to reach the elimination goals? Liver Int. 2018;38(12):2190-8. https://doi.org/10. 1111/liv.13901.

8. Direzione e Redazione Presso il Ministero della Giustizia - Ufficio Pubblicazione Leggi e Decreti, "Supplemento ordinario alla 'Gazzetta Ufficiale' n. 51 del 29 febbraio 2020 - Legge N. 8 Articolo 25 'Screening nazionale gratuito per l'eliminazione del virus HCV.'”. https://www.gazzettaufficiale.it/eli/gu/2020/02/29/51/so/ 10/sg/pdf. Accessed 22 Dec 2020.

9. Mennini FS, et al. The impact of direct acting antivirals on hepatitis $\mathrm{C}$ virus disease burden and associated costs in four European countries. Liver Int. 2021;41(5):934-48.

10. Thein HH, Yi Q, Dore GJ, Krahn MD. Estimation of stage-specific fibrosis progression rates in chronic hepatitis $\mathrm{C}$ virus infection: a meta-analysis and meta-regression. Hepatology. 2008;48(2):41831. https://doi.org/10.1002/hep.22375.

11. Dienstag JL, et al. A prospective study of the rate of progression in compensated, histologically advanced chronic hepatitis C. Hepatology. 2011;54(2):396-405. https://doi.org/10.1002/hep.24370.

12. Wright M, Grieve R, Roberts J, Main J, Thomas HC, UK Mild Hepatitis C Trial Investigators. Health benefits of antiviral therapy for mild chronic hepatitis $\mathrm{C}$ : randomised controlled trial and economic evaluation. Health Technol Assess. 2006;10(21):1-113. https://doi.org/10.3310/hta10210.

13. Morgan RL, Baack B, Smith BD, Yartel A, Pitasi M, Falck-Ytter Y. Eradication of hepatitis $C$ virus infection and the development of hepatocellular carcinoma: a meta-analysis of observational studies. Ann Intern Med. 2013;158(1):329-37. https://doi.org/ 10.7326/0003-4819-158-5-201303050-00005.

14. Progetto PITER - Piattaforma Italiana per lo studio della Terapia delle Epatiti viRali. https://www.progettopiter.it/. Accessed 10 Feb 2020.

15. Kondili LA, Andreoni M, Alberti A, et al. Estimated prevalence of undiagnosed hepatitis $\mathrm{C}$ virus infected individuals in Italy: a mathematic model to accurately measure HCV prevalence with a route of transmission granularity. Epidemics. 2020;34: 100442. https://doi.org/10.1016/j.epidem.2021.100442.

16. StatisticsIEurostat|Life table. 2018. https://ec.europa.eu/euros tat/databrowser/view/demo_mlifetable/default/table?lang=en. Accessed 20 Oct 2020.

17. Briggs A, Sculpher M, Claxton K. Decision modelling for health economic evaluation. Oxford: Oxford University Press; 2006.

18. Linthicum MT, et al. Value of expanding HCV screening and treatment policies in the United States. Am J Manag Care. 2016;22(6):227-35

19. Razavi H, Sanchez-Gonzalez Y, Yuen C, Cornberg M. Global timing of hepatitis $\mathrm{C}$ virus elimination in high-income countries. Liver Int. 2020;40(3):522-9. https://doi.org/10.1111/liv.14324.

20. Polaris Observatory-CDA Foundation. https://cdafound.org/ polaris/. Accessed 22 Dec 2020

21. Kondili LA, et al. Optimization of hepatitis $\mathrm{C}$ virus screening strategies by birth cohort in Italy. Liver Int. 2020;40(7):1545-55. https://doi.org/10.1111/liv.14408.

22. Kondili LA, Blach S, Razavi H, Craxi A. Tailored screening and dedicated funding for direct acting antiviral drugs: how to keep Italy on the road to hepatitis $C$ virus elimination? Ann Ist Super Sanità. 2020;56(3):325-9. https://doi.org/10.4415/ANN_20_03_10.

23. Kondili LA, Vella S. PITER: an ongoing nationwide study on the real-life impact of direct acting antiviral-based treatment for chronic hepatitis C in Italy. Dig Liver Dis. 2015;47:741-3. https:// doi.org/10.1016/j.dld.2015.05.022. 